Physics

Electricity \& Magnetism fields

Okayama University

Year 1992

\title{
A simple algorithm for adaptive refinement of tetrahedral meshes combined with edge elements
}

\author{
Piotr Olszewski \\ Okayama University \\ Norio Takahashi \\ Okayama University
}

\author{
Takayoshi Nakata \\ Okayama University \\ Koji Fujiwara \\ Okayama University
}

This paper is posted at eScholarship@OUDIR : Okayama University Digital Information Repository.

http://escholarship.lib.okayama-u.ac.jp/electricity_and_magnetism/148 


\title{
A Simple Algorithm for Adaptive Refinement of Tetrahedral Meshes Combined with Edge Elements
}

\author{
P. Olszewski, T. Nakata, N. Takahashi and K. Fujiwara
}

Department of Electrical Engineering, Okayama University, Okayama 700, Japan

\begin{abstract}
This paper presents a simple algorithm yielding unique face subdivision patterns of locally refined tetrahedral elements by the Delaunay tessellation process. Due to numerous advantages of edge elements, the algorithm is combined with the magnetostatic edge element computer code. Next, a comparative analysis of four different error estimates used to locate elements for refinement, including a new one suitable for edge elements, is described. Finally, the performance of the presented algorithm and the effectiveness of the error estimates are demonstrated by means of three-dimensional test problems.
\end{abstract}

\section{INTRODUCTION}

Among various methods proposed recently for mesh generation and refinement in finite element analysis, the majority are based on the Delaunay tessellation applied globally, to all elements in the mesh (see e.g. $[1,2])$. The serious disadvantage of the Delaunay optimization process applied to the whole mesh is greater than linear time complexity [3]. A more efficient approach, recently described in [4], is to confine the number of the processed elements to only those having excessive errors, and perform the refinement in each of them locally, one-by-one. The primary difficulty of this technique not discussed in [4], is maintaining compatibility between individually subdivided tetrahedrons. For that purpose, an algorithm that controls the refinement so that a match of faces for each two refined elements can be reached at their common face, is required. Such an algorithm based on the ordering of new points for the Delaunay tessellation according to the lengths of the element edges is described.

As argued in [5-7], the choice of discrete variables associated with edges rather than nodes is more correct for approximation of gradient fields, and edge elements provide more accurate and cheaper solutions. Therefore, the discussed algorithm is coupled with a finite element magnetostatic formulation using the magnetic vector potential and first order edge elements.

Four different a posteriori error estimates that indicate elements to be refined are tested with the present refinement scheme. The first three estimates utilize the discontinuity of the tangential component of the magnetic field at element interfaces $[4,8,9]$. The third estimate, presented here for the first time, is designed to generate uniform refinements, independently of the tree selected, when the co-tree technique is used to impose the gauge condition [10]. The fourth, previously used in elasticity [11], is based on a projection process that provides a $\mathrm{Cl}^{1}$ approximation of the field intensity vector inside elements using a $\mathrm{C}^{0}$ approximation obtained by calculating the rotation of the magnetic vector potential. The difference between the two approximated solutions $\left(\mathrm{C}^{1}\right.$ and $\left.\mathrm{C}^{0}\right)$ integrated over the problem region is used as a measure of the smoothness of the solution. This measure is independent of the error estimation method and constitutes itself a convenient indicator for the convergence criterion of the adaptive refinement iteration process.

\section{MESH REFINEMENT ALGORITHM}

The local Delaunay tessellation of octants in terms of octree/ Delaunay three-dimensional mesh generation has been extensively investigated in [3]. Octant incompatibility due to two-dimensional degeneracies on octant faces is prevented by ordering the octant points in the Delaunay tessellation. A similar method is used in our algorithm with reference to locally refined tetrahedrons. The new points for the Delaunay algorithm are located in the middle of edges, whose vertices (one or two) have excessive nodal errors, as shown in Fig. 1. Thus, similar to [4], in a tetrahedron having one excessive vertex, three midpoints are set. Two selected vertices generate five new mid-points, and three or four vertices above the acceptable error level form six new points. Performing refinement according to the above rule reduces the number of possible face subdivision patterns, to only the three cases, shown in Fig. 1, and thus considerably simplifies the difficulty in handling the face compatibility between two refined elements, as Manuscript received June 1, 1992, revised September 4 1992. This work was
upported in part by the Grant-in-Aid for Co-operative Research(A) from the supported in part by the Grant-in-Aid for Co-operative Research $(A)$
Ministry of Education, Science and Culture in Japan (No. 01302031).

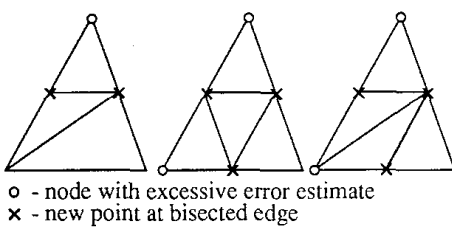

Fig. 1. Face subdivision patterns.

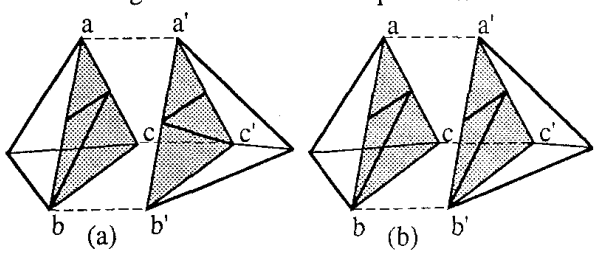

Fig. 2. Elements: (a) Non-compatible; (b) Compatible.

shown in Fig. 2

In the majority of cases, the ordering of new points according to the lengths of element edges forms a unique Delaunay tessellation that guarantees compatibility. Either a descending or ascending order may be applied. Mid-points located at edges having equal lengths are ordered according to edge global numbers. When an element is refined, the face pattern types shown in Fig. 1 are assigned to each face, and next, the compatibility test that compares face patterns of the actual element with the corresponding face patterns of four adjacent elements is made. Although occurrence of the "crossed edges" type case is very rare (e.g. none in both of the test problems described in Section IV), this however must be resolved in order to obtain a reliable meshing algorithm. In several cases, like the one in Fig. 3, the compatibility is ensured by rearranging the order of new points. Note the unchanged subdivisions of the remaining faces. This may be applied to previously or actually processed elements.
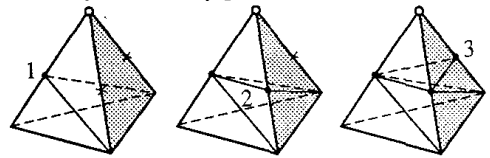

(a) order 1-2-3
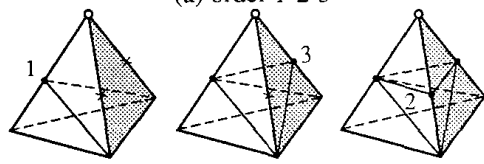

(b) order 1-3-2

Fig. 3. Modifying subdivision of dotted face by changing the order of the new points.

When the swapping of edges according to this scheme is not possible or fails to resolve the degenerate case, then the following is applied. If all four neighbors of the actual element are already refined, a new node is added at the center of gravity, and all face triangles are connected to it. If one or more neighbors have not yet been refined, then the face patterns are unknown and the element is flagged for processing till all neighbors are refined. If subdivisions for two adjacent elements cannot be formed, an arbitrary face pattern from the set shown in Fig. 1 is assigned to their common face. The resulting mesh is locally a Delaunay tessellation, apart from the elements with added mid-nodes.

\section{ERROR ESTIMATES}

When magnetostatic problems are described in terms of the magnetic vector potential $\mathbf{A}$ by the equation

$$
\operatorname{curl}(v \operatorname{curl} \mathbf{A})=\mathbf{J}
$$


and linear edge interpolation is used to approximate the vector function over elements, then the field $\mathbf{A}$ is tangentially continuous at all element interfaces $[5,6]$. Since the normal component of $\mathbf{A}$ is not directly represented, both tangential components of the flux density and the field intensity may be discontinuous across interfaces. However, the physical fields $\mathbf{B}$ and $\mathbf{H}$ are required by Maxwell's equations to be continuous everywhere apart from boundaries between materials of different magnetic reluctivity. Thus, the surface current density $\mathbf{K}$ resulting from the difference of the tangential components of the field intensity $\mathbf{H}_{t}$ at both sides of the interface constitutes a convenient error estimate $e_{l}$ of the local error introduced by the numerical solution

$$
e_{l}=\mathbf{K}=\left(\mathbf{H}_{1}-\mathbf{H}_{2}\right) \times \mathbf{n}_{12},
$$

where $\mathbf{n}_{12}$ is a normal vector to the interface, directed from element 1 to element 2 .

When the vector $\mathbf{K}$ is weighted by the vector potential $\mathbf{A}$ over the interface surface $S$, a global estimate $e_{2 i}{ }^{\prime}$ is formed [4],

$$
e_{2 i}^{\prime}=\frac{1}{2} \int_{S} \mathbf{K} \cdot \mathbf{A} d S
$$

that can distinguish between regions of large discontinuities of the tangential field $\mathbf{H}_{\mathrm{t}}$ and significant values of the potential $\mathbf{A}$. Equation (3) reveals that only the component $\mathbf{A}_{t}$ contributes to the estimate, because the normal component $\mathbf{A}_{\boldsymbol{n}}$ is orthogonal to $\mathbf{K}$ at every point of the interface. Thus, for the uniqueness of the estimate $e_{2 i}$ at both sides of the interface, the continuity of $\mathbf{A}_{t}$ across element interfaces is necessary. This condition is precisely satisfied by edge elements. The modified error estimate $e_{2 I}$ is given by

$$
e_{2 I}=\frac{1}{2} \int_{S} \mathbf{K} \cdot \mathbf{A}_{\mathrm{t}} d S .
$$

For magnetostatic problems described by (1), Neumann boundary conditions are enforced naturally and the tangential field intensity $\mathbf{H}_{t}$ is not everywhere equal to zero at Neumann boundaries. Then, the error contributions from faces embedded in Neumann boundaries may be expressed as

$$
e_{2 N}=\frac{1}{2} \int_{S} \mathbf{H}_{\mathrm{t}} \cdot \mathbf{A}_{\mathrm{t}} d S .
$$

When the co-tree strategy described in [10] is applied to impose the gauge condition, then the spatial distribution of the field $\mathbf{A}$ varies from one tree to another, and so does the refinement, when (4) and (4') are used for error estimation. The way to eliminate this ambiguity is to use the normal component of the flux density $\mathbf{B}$, instead of $\mathbf{A}_{t}$. This can be done because the choice of the tree does not affect the $\mathbf{B}$ solution and the continuity of $\mathbf{A}_{\mathrm{t}}$ implies that of $\mathbf{B}_{\mathrm{n}}$. By analogy to (4) and (4'), the new error estimate is

$$
\begin{aligned}
& e_{3 I}=|\mathbf{K}| \cdot\left|\mathbf{B}_{\mathbf{n}}\right| \cdot S=|\mathbf{K}| \cdot \Phi \\
& e_{3 N}=\left|\mathbf{H}_{\mathbf{t}}\right| \cdot\left|\mathbf{B}_{\mathrm{n}}\right| \cdot S=\left|\mathbf{H}_{\mathbf{t}}\right| \cdot \Phi,
\end{aligned}
$$

where $\Phi$ is the magnetic flux crossing the interface $S$.

To explain the projection process used to obtain the fourth error estimate $e_{4}$, we assume for the moment an isotropic, single medium, two-dimensional case and linear approximation $\mathbf{A}^{1}=\mathbf{N} \underline{\mathbf{A}}^{1}$ of the vector potential $\mathbf{A}$ in (1). $\mathbf{N}$ here stands for nodal shape functions, $\mathbf{A}^{1}$ denotes potential values at nodes and superscript $1, \mathrm{C}^{1}$ continuity. In such a case, the potential A has only one spatial component, say z. A linear approximation of $\mathbf{A}_{\mathbf{z}}$ results in a discontinuous approximation of $\mathbf{B}$ and $\mathbf{H}$, as illustrated schematically in Fig. 4 for the $x$-component of $\mathbf{H}^{0}$ along the direction parallel to $y$-axis. Superscript 0 denotes $\mathrm{C}^{0}$ continuity of $\mathbf{H}$. Now, calculating the projection

$$
\int_{\Omega} \mathbf{N}^{\mathrm{T}}\left(\mathbf{H}^{1}-\mathbf{H}^{0}\right) d \boldsymbol{\Omega}=0,
$$

in which it is assumed that the field $\mathbf{H}$ is interpolated in the region $\Omega$ by the same function as the potential $\mathbf{A}$,

$$
\mathbf{H}^{1}=\mathbf{N} \underline{\mathbf{H}}^{1}
$$

a smoothed field $\mathbf{H}^{1}$ can be found, as shown for the $x$-component of $\mathbf{H}^{1}$ in Fig. 4. As explained in [11], the field $\mathbf{H}^{1}$ is a better approximation than the field $\mathbf{H}^{0}$. Therefore, we shall use a half of the square of the difference between the two approximated solutions integrated over element volume $\Omega^{(e)}$ as an element error estimate $e_{4}$

$$
e^{4}=\frac{1}{2 \mathbf{V}^{(e)}} \int_{\Omega^{(e)}}\left(\mathbf{H}^{1}-\mathbf{H}^{0}\right)^{2} d \Omega^{(e)} .
$$
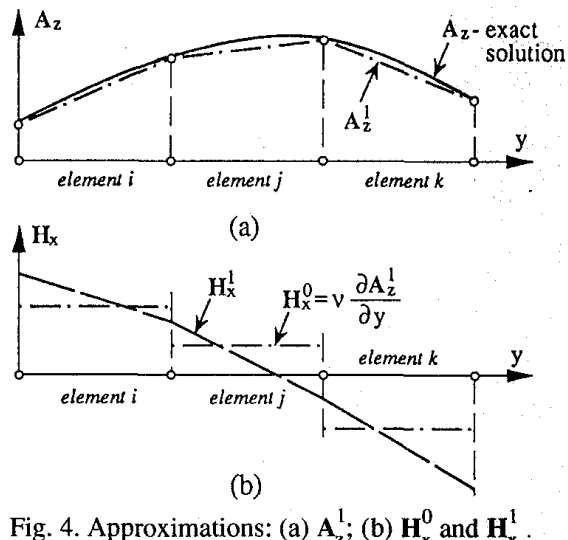

which is given the unit of energy when multiplied by the element reluctivity $v^{(e)}$. By choice of edge shape functions, problems with more than one material may be approached because discontinuities across interfaces are automatically taken into account. From (6), (7) and $\mathbf{H}^{0}=v \nabla \times \mathbf{A}=\nu \nabla \times\left(\mathbf{N A}^{1}\right)$ in terms of edge values of the vector potential, the projection $\underline{\mathbf{H}}^{1}$ can be obtained from

$$
\underline{\mathbf{H}}^{1}=\mathbf{U}^{-1} \int_{\Omega} v \mathbf{N}^{\mathrm{T}} \nabla \times\left(\mathbf{N} \underline{\mathbf{A}}^{1}\right) d \Omega
$$

where

$$
\mathbf{U}=\int_{\Omega} \mathbf{N}^{\mathrm{T}} \mathbf{N} d \Omega
$$

By using estimate $e_{4}$, the square of the error norm over the whole domain may be calculated as

$$
\|\mathbf{e}\|^{2}=\sum_{i=1}^{m}\left(e_{4 i}\right)^{2},
$$

where $i$ represents an element contribution and $m$ the total element number. The global smoothness of the solution may be assessed by the relative percentage error $\eta$ of the form [11]:

$$
\eta=\left(\|\mathbf{e}\|^{2} /\left(\|\mathbf{e}\|^{2}+E^{2}\right)\right)^{1 / 2} \cdot 100 \% .
$$

where $E$ is the energy stored in the system. The smoothness of the solution measured by the error $\eta$ may be calculated for any error estimate. Therefore, $\eta$ establishes a convenient tool to evaluate the effectiveness of various estimates and can be used to terminate the adaptive refinement iteration process when desired by the user smoothness of the solution is achieved. For both test problems described in Section IV, refinement stops when $\eta \leq 2 \%$ (see Fig. 9.)

Nodal errors at element vertices required by the refinement algorithm are calculated in the following way. For the first estimation method, the largest magnitude of the error $e_{1}$ of all faces sharing a given node is assumed. For the second and third estimation methods at first one third of the sum of contributions $e_{2 I}$ or $e_{3 I}$ from all interfaces common to a given node are taken. Next, nodal errors of nodes located at Neumann boundaries are doubled so that the contributions from regions behind the Neumann boundaries are also considered, and finally one third of facial contributions $e_{2 N}$ or $e_{3 N}$ are added to each Neumann node. The fourth estimation method yields errors in elements and not interfaces. In each element, one quarter of $e_{4}$ is assigned to every node, and for all nodes contribuions from all elements sharing a given node are summed up at that node. Then, the resultant nodal error is averaged by the number of the contributing elements. To compare the performances of the four estimators, for each method the same percentage $(30 \%)$ of the nodes with the most error is selected at each refinement step as above the permissible error level.

\section{ILLUSTRATIVE EXAMPLES}

Two magnetostatic test problems: uniform current in a bus-bar and uniform current in a cube, are chosen as examples. The first problem is used to show the meshing process at four successive refinement steps. The second is used to test the performance of the four estimators described in the previous section. Although the two problems are of two-dimensional character, they are studied here as fully threedimensional ones. Both magnetic fields are induced in vacuum by a current having the same direction and density equal to $J_{y}=10000$ 
$\mathrm{A} / \mathrm{m}^{2}$. For both cases, the starting mesh for a new iteration step is the one used to obtain the solution at the previous iteration, except for the initial step. The two problems have analytical solutions, that enable comparison between various numerical solutions.

\section{A. Uniform current in a bus-bar}

The unit length of one meter of an infinitely long bus-bar having $0.19 \times 0.19 \mathrm{~m}$ cross section, is analyzed. The bar is enclosed in a finite region and due to symmetry, only one quarter is considered, as shown in Fig. 5. Homogeneous Dirichlet boundary conditions $\mathbf{A}_{t}=0$ are set to all boundaries except for the $x=0$ and $z=0$ symmetry planes, these being the Neumann boundaries of the problem domain. The initial mesh consists of 450 tetrahedral elements. The second estimate $\left(e_{2 I}\right.$ and $e_{2 N}$ given by (4) and $\left(4^{\prime}\right)$ ) was assumed for computations. Symmetry and the two-dimensional character of this model simplifies the visualization of the meshing. For that purpose subdivisions at two control planes: one internal at $y=0.333 \mathrm{~m}$, and the other boundary at $x=0$, marked by the dashed line in Fig. 5 , are chosen.

The characteristic of the meshing of the initial and four refinement steps are shown in Figs. 5 and 6. For clarity, the initial mesh at the $x=0$ plane is moved backwards in Fig. 5. The figures show that refinement concentrates inside the bar and in close proximity to it. This is in good agreement with the local character of the magnetic field, because the field decays logarithmically with the distance from the $y$ axis. The results of the field computations are shown in Fig. 7. Comparison with the exact solution justifies the need for considerable meshing readily carried out by the algorithm in the proximity of the bar where the field changes rapidly. Quick convergence of the nodal estimate in the whole domain is shown in Fig. 8. The graph is obtained by computing the percentage of nodes falling into ten, equally spaced intervals into which the logarithmic range of nodal errors is split. As refinement proceeds, the errors become small and uniformly distributed over the problem domain.

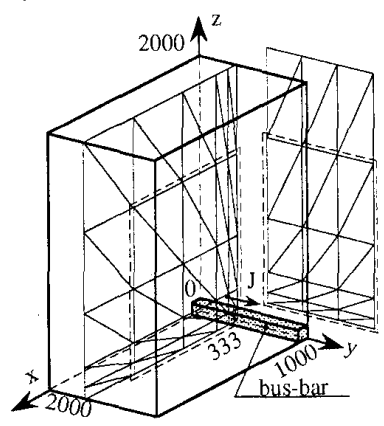

Fig. 5. First test problem and initial subdivisions on two planes.

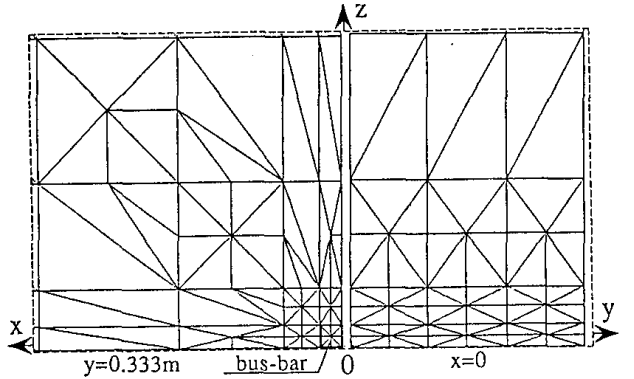

(a) ist refinement (1356 elements)

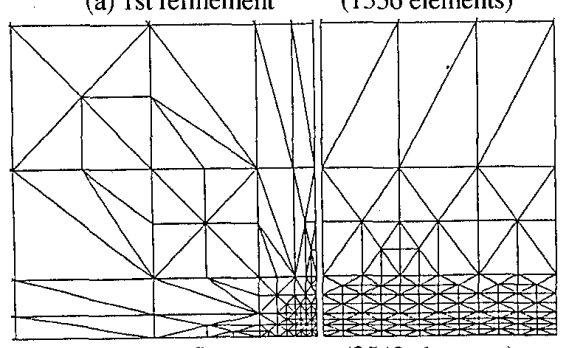

(b) 2nd refinement

(3540 elements)

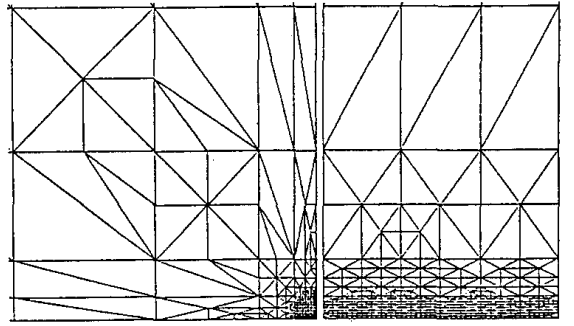

(c) 3rd refinement
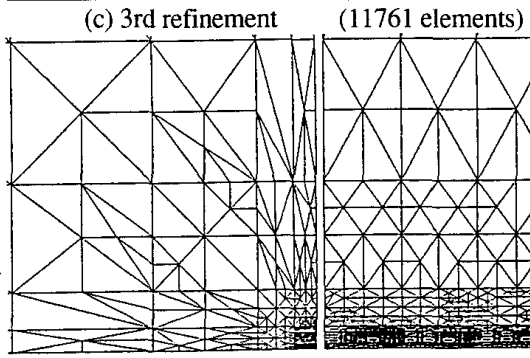

(d) 4th refinement

(49374 elements)

Fig. 6. Meshing for four successive refinement steps.

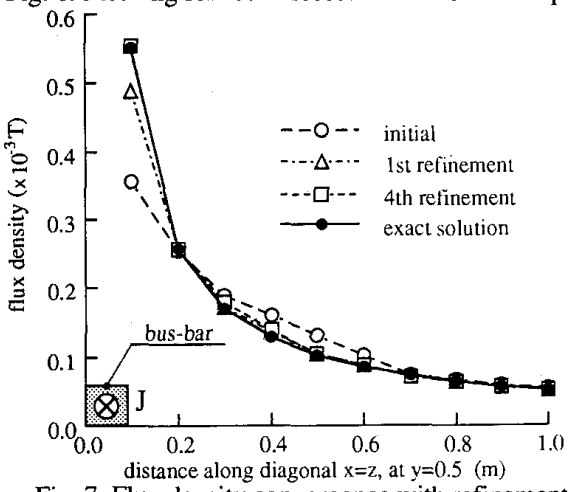

Fig. 7. Flux density convergence with refinement.

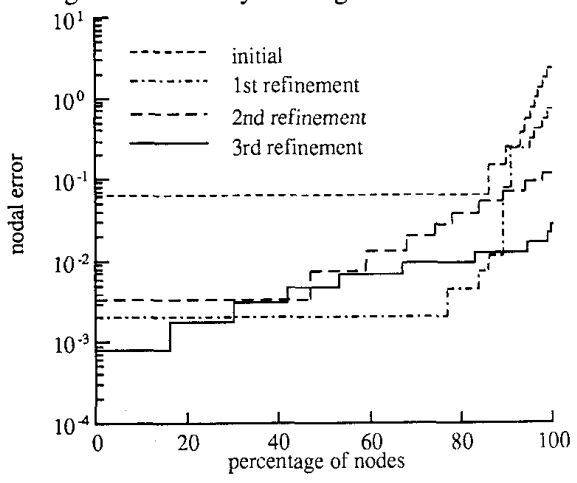

Fig. 8. Convergence of nodal errors with refinement.

\section{B. Uniform current in a cube}

The problem domain is a cube, having each side one meter long [6]. Homogeneous Dirichlet boundary conditions are imposed at boundaries. For each estimate, the initial mesh was the same and consisted of 48 tetrahedrons. The performance is shown in Figs. 9, 10 and 11 , in terms of the relative percentage error $\eta$, mesh quality measured by the aspect ratio and the total energy stored in the system. The aspect ratio is defined as the quotient of the circumscribed sphere over the radius of the inscribed sphere. In these graphs, we refer to the four estimates as: the $\mathbf{K}$ estimate $\left(e_{1}\right.$ given by (2)), $\mathbf{K}$ weighted by $\mathbf{A}_{\mathrm{t}}\left(e_{2 I}\right.$ and $e_{2 N}$ given by (4) and (4)), $\mathbf{K}$ weighted by $\mathbf{B}_{\mathrm{n}}\left(e_{3 I}\right.$ and $e_{3 N}$ given by $(5)$ and $\left.\left(5^{\prime}\right)\right)$ and the projection estimate $\left(e_{4}\right.$ given by $(8)$ ), respectively. All graphs are plotted against the number of un- 


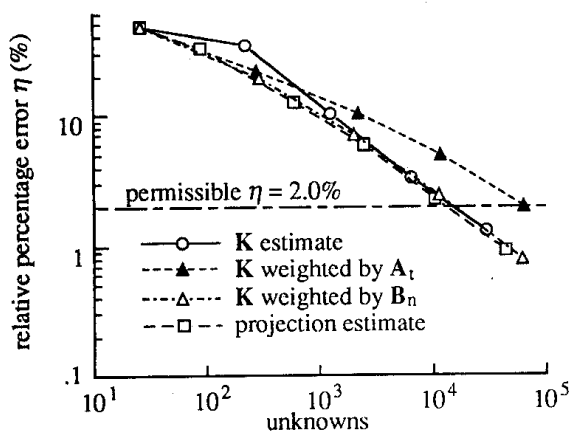

Fig. 9. Rate of convergence of error $\eta$.

knowns. In case of Fig. 9, we may observe the same convergence rate of the relative error $\eta$ for all estimates apart from the "K weighted by $\mathbf{A}_{\mathrm{t}}$ "-type, that converges more slowly. Fig. 10 shows that the quality of the initial mesh is maintained throughout the refinement because for all estimation methods the average aspect ratio is virtually invariant. At the final refinement, the maximum aspect ratios for the two first estimators are larger, more than twice, compared with the two others which produce more regular meshes. Also the convergence to the exact value of the stored energy which in this case is $2.208 \mathrm{~J}$, is very good for the " $K$ weighted by $B_{n}$ "-type and poor for the " $K$ weighted by $\mathbf{A}_{t}$ "-estimator, as shown in Fig. 11. Small differences were observed in the case of the CPU time that varies linearly with the unknowns with somewhat faster performance for the $\mathbf{K}$-type estimate.

The discussed problem calls for refinement distributed uniformly along $y$-axis and concentrated towards boundaries, especially at the corners. Such refinement is obtained when the "K weighted by $B_{n}$ "type estimator is used. Since the field $\mathbf{A}$ decreases to zero near the boundaries due to the imposed boundary conditions, the " $K$ weighted by $A_{t}{ }^{\prime \prime}$-type estimator cannot be equally efficient. In such cases the new " $K$ weighted by $B_{n}$ "-estimator is clearly advantageous.

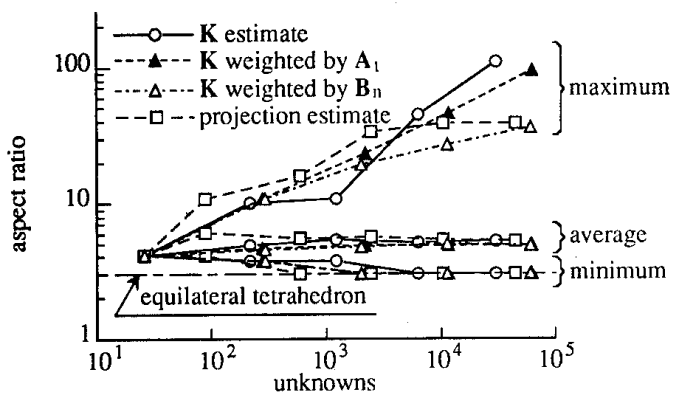

Fig. 10. Quality of meshing.

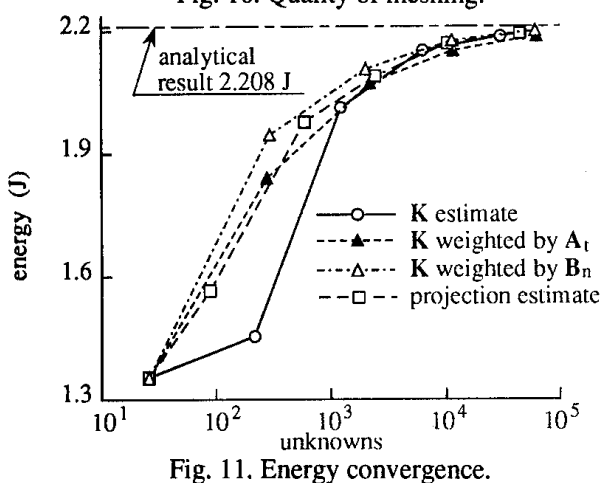

\section{CONCLUSIONS}

A simple algorithm yielding unique triangulations of tetrahedral faces when the Delaunay tessellation process is applied to refine elements locally, is presented. A new efficient error estimate suitable for use together with edge elements, is described. The projection method described in the paper, is shown to provide a convenient indicator that can be used independently of the error estimation method applied to terminate the refinement process when the desired smoothness of the solutions is attained. Finally, by means of two simple test problems the meshing performed by the described algorithm is demonstrated and the performance of four different error estimators is evaluated.

\section{REFERENCES:}

[1] J.C. Cavendish, D.A. Field and W.H. Frey, “ An approach to automatic three-dimensional finite element mesh generation," Int. J. Num. Meth. Eng., vol. 29 , pp. 329-347, 1985.

[2] D.N. Shenton and Z.J. Cendes, "Three-dimensional finite element mesh generation using Delaunay tessellation," IEEE Trans. Magn., vol. 21 , No. 6 pp. 2535-2538, November 1985.

[3] W.J. Shroeder and M.S. Shephard, " A combined octree/Delaunay method for fully automatic 3-D mesh generation," Int. J. Num. Meth. Eng., vol. 29, pp. $37-55,1990$.

[4] T.W. Nehl and D.A. Field, " Adaptive refinement of first order tetrahedral meshes for magnetostatics using local Delaunay subdivisions," IEEE Trans. Magn., vol. 27, No. 5, pp. 4193-4196, September 1991.

[5] A. Bossavit, "Whitney forms: a class of finite elements for three dimensional computations in electromagnetics," IEE Proc., vol. 135, Pt. A pp. $457-462,1988$

[6] M.L. Barton and Z.J. Cendes, "New vector finite elements for threedimensional magnetic field computation," $J$. Appl. Phys. vol. 61, No. 8, pp 3919-3921, 15 April 1987

[7] T. Nakata, N. Takahashi, K. Fujiwara and P. Olszewski, " Solution of TEAM workshop problem 13 using edge elements and the magnetic vector potential," Digest of the 14th Annual Conference on Magnetics in Japan, $\mathrm{p}$ 393, October 1990

[8] H. Kim, S. Hong, K. Choi and S. Hahn, " A three dimensional adaptive finite element method for magnetostatic problems," IEEE Trans. Magn., vol. 27, No. 5, pp. 4081-4084, September 1991.

[9] S. Ratnajeevan H. Hoole, S. Yoganathan and S. Jayakumaran "Implementing the smoothness criterion in adaptive meshes," IEEE Trans. Magn., vol. 22, No. 5, pp. 808-810, September 1986.

[10] R. Albanese and G. Rubinacci, " Magnetostatic field computations in terms of two-component vector potentials," Int. J. Num. Meth. Eng, vol. 29, pp: 515-532, 1990

[11] O.C. Zienkiewicz and J.Z. Zhu, " A simple error estimate and adaptive procedure for practical engineering analysis," Int. J. Num. Meth Eng., vol. 24, pp. 337-357, 1987.

Piotr Olszewski was born in Lodz, Poland, in 1958. He received his Dipl Eng. degree from the Department of Electrical Engineering at the Technica University of Lodz, Lodz, Poland, in 1984. From 1984 to 1988 he worked as a research assistant at the Institute of Electrical Machines and Transformers, the Technical University of Lodz. In 1988 he was awarded a research scholarship by the Japanese Ministry of Education and in 1990 began his postgraduate study at Okayama University, Okayama, Japan. His current interest is the application of the finite element method to various engineering problems.

Takayoshi Nakata (M'72-SM'91) was born in Ehime prefecture, Japan, in 1930 and received the B.E. and Ph.D. degrees in electrical engineering from Kyoto University in 1953 and 1971, respectively. From 1953 to 1962 , he was with Fuji Electric Manufacturing Co. Ltd., where he was engaged as a design and development engineer of large transformers. Since 1963, he has been a Professor at the Department of Electrical Engineering, Okayama University. His major fields of interest are the applications of the finite element method to electrical machines and electronic instruments, magnetic characteristics of transformer cores and manetic electronic int measurd Award and the of Japan. He established the IEEE Magnetics Society's Tokyo Chapter in 1977 ference and a member of the working group on recommended practices for field computation and analysis in electric machinery of the IEEE Power Engineering Society.

Norio Takahashi (M'87) was born in Hyogo Prefecture, Japan, in 1951. He received the B.E. degree in electrical engineering from Okayama University in 1974 and the M.E. and Ph.D. degrees in electrical engineering from Kyoto University in 1976 and 1982, respectively. Since 1984, he has been an Associate University in 1976 and 1982 , respectively. Since 1984, he has been an Associate Professor at the Department of Electrical Engineering, Okayama University. His major interests are development of numerical methods for calculating magnetic
fields, forces and torques, and optimal design method for magnetic devices fields, forces and torques, and optimal design method for magnetic devices. Dr.Takahashi was awarded the 1983 Book Award of the Institute of Electrica
Engineers of Japan. He is a vice-chairman of TEAM Workshop Planning Board.

Koji Fujiwara (M'90) was born in Hiroshima Prefecture, Japan in 1960. He received the B.E. and M.E. degrees in electrical engineering from Okayama University in 1982 and 1984 , respectively. From 1985 to 1986 , he was with Mitsui Engineering and Shipbuilding Co., Ltd. Since. 1986, he has been an Assistant Professor at the Department of Electrical Engineering, Okayama University. His major fields of interest are applications of the 3-D finite element method to electromagnetic field calculation and acceleration of solvers of large scale simultaneous equation. 